\title{
Ground and Excited State Dipole Moment of 2, 5-Dihydroxybenzoic Acid from Solvatochromic Shifts
}

\author{
Meena Adhikari and Sanjay pant \\ Photophysics Laboratory, Department of Physics (UGC-Centre of Advanced Study), Kumaun University, \\ Nainital \\ Email: moonaadhikari03@gmail.com

\begin{abstract}
In present work we explore the role of solvents on the 2, 5-dihydroxybenzoic acid by using fluorescence techniques. It was found that DHBA shows spectral behaviour depending on polarity of solvents. DHBA shows generally three components at 405-415nm, 430-450nm, and 450-470nm. The effects of zwitterion as well as the interplay ionic species are involved to explain the observed results.
\end{abstract}

Keywords: Fluorescence techniques, Dipole moments, DHBA.

\section{Introduction}

Solvatocromic shifts are fundamental experimental observation which are of importance for the photophysical and photochemical properties of the molecules in the various solvents [3]. A change in solvent is accompanied by a change in polarity, dielectric constant or polarizability of surrounding medium [4]. Thus the change of solvent affects the ground and excited state differently [5].The loss of energy i.e. Stokes shift, between the excitation and deexcitation of the fluorophore is a result of several dynamic processes which include dissipation of vibrational energy, redistribution of electrons induced by the increases of the excited state dipole moment of fluorophore and interaction of solvent molecule with the solute or fluorophore [6]. To the first approximation this energy difference (in $\mathrm{cm}^{-}$ ${ }^{1}$ ) is the property of the refractive index $(\eta)$ and dielectric constant $(\varepsilon)$ of the solvent and is described by the equation.

\begin{abstract}
$v_{\mathrm{a}}-v_{\mathrm{f}}=\frac{2\left(\mu_{\mathrm{e}}-\mu_{g}\right)^{2}}{h \sigma a^{\mathrm{s}}}\left(\frac{2 \eta^{2}+1}{\eta^{2}+1}\right)\left[\frac{\mathrm{s}-1}{\mathrm{~s}+1}-\frac{\eta^{2}-1}{\eta^{2}+2}\right]+$ constant

Where $v_{\mathrm{a}}$ and $v_{\mathrm{f}}$ (in $\mathrm{cm}^{-1}$ ) are the wavenumbers of absorption and emission respectively, $\mathrm{h}$ is a plank constant, $\mathrm{c}$ is a speed of light, $\eta$ is a refractive index and a is a radius of the cavity in which the fluorophore resides.
\end{abstract}

\section{Experimentation Material}

2, 5-Dihydroxybenzoic acid was purchased from Aldrich of $98 \%$ purity and used as such. All the solvents used were either of HPLC or spectroscopic grades. Freshly prepared samples at room temperature were used in the present study. The concentration of DHBA was kept at $1 \times 10^{-4} \mathrm{~mol} / \mathrm{L}$. in solvent study.

\section{Instrumentation}

Steady state absorption spectra were recorded with the help of UV-Visible Double beam 
Systronics Spectrophotometer. At ambient temperature $(296 \mathrm{~K})$, the steady state electronic absorption spectra of dilute solution $\left(10^{-5} \mathrm{M}\right)$ of all samples, were recorded. The emission and excitation spectra were recorded by using jobinfluorolog YVON FL3-22.

\section{Methods}

Generally following expression are used for the estimation of dipole moment and stoke shift.

$v_{a}-v_{f}=S_{1} f_{1}(\varepsilon, \eta)+$ constant
$\frac{\text { va+ vf }}{2}=S_{2} f_{2}(\varepsilon, \eta)+$ constant

Where $v_{\mathrm{a}}$ and $v_{\mathrm{f}}$ are absorption and fluorescence maxima wavelength in $\mathrm{cm}^{-1}$, respectively.

And $S_{1}=\frac{2\left(\mu_{e}-\mu_{g}\right)^{2}}{h e a^{g}}, S_{2}=\frac{2\left(\mu_{g}{ }^{2}-\mu_{g}\right)^{2}}{h e a^{g}}$

$\mu_{g}$ and $\mu_{e}$ are the ground and excited state dipole moments of the solute molecule .The symbols $\mathrm{h}$ and $\mathrm{c}$ are Planck's constant and velocity of light in vacuum respectively, 'a' is the Onsager radius of the solute molecule.The parameter $S_{1}$ and $S_{2}$ can be obtained from the absorption and fluorescence band shifts. The slopes which can be calculated using equation (1) and (2) respectively.

\section{Results and discussion}

The absorption and fluorescence spectra of 2,5-dihydroxybenzoic acid were recorded in polar, polar aprotic and non polar solvents in ambient temperature and are shown in Fig. 2 and Fig. 3.

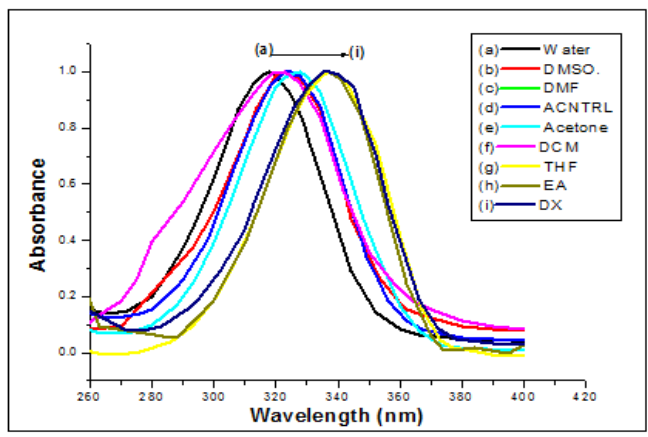

Fig. 2 Absorption of 2,5-DHBA in different solvents

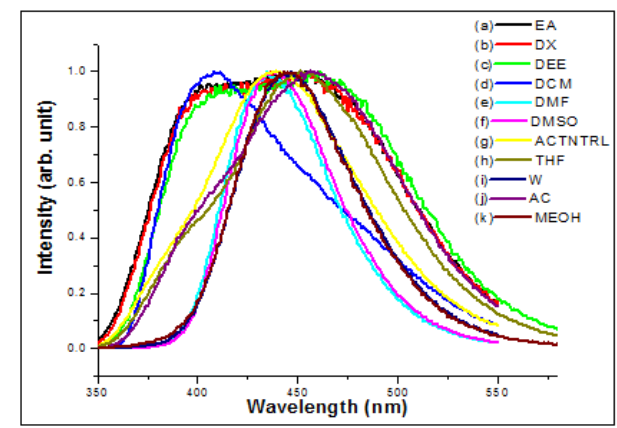

Fig. 3 Emission spectra of 2,5-DHBA in different solvents

\begin{tabular}{llllll}
\hline Solvents & $\eta$ & $\mathbf{F}_{\mathbf{1}}$ & $\mathbf{F}_{\mathbf{2}}$ & $\mathbf{v}_{\mathbf{f}}-\mathbf{v}_{\mathbf{a}}\left(\mathbf{c m}^{-\mathbf{1}}\right)$ & $\frac{\mathbf{v}_{\mathbf{z}}+\mathbf{v}_{\mathbf{f}}}{\mathbf{2}}\left(\mathbf{c m}^{-\mathbf{1}}\right)$ \\
\hline Water & 1.33 & 0.91 & 0.68 & 8924.02 & 26984.53 \\
Methanol & 1.32 & 0.85 & 0.65 & 7965.28 & 26505.16 \\
Propanol & 1.38 & 0.78 & 0.65 & 7881.51 & 26362.27 \\
Butanol & 1.39 & 0.75 & 0.64 & 7698.96 & 26271 \\
Heptanol & 1.42 & 0.50 & 0.53 & 7677.8 & 26011.84 \\
Octanol & 1.42 & 0.23 & 0.40 & 7677.8 & 26011.84 \\
DMSO & 1.42 & 0.84 & 0.67 & 8076.46 & 26921.52 \\
DMF & 1.43 & 0.71 & 0.83 & 26974.12 & 7971.25 \\
ACN & 1.32 & 0.86 & 0.65 & 26774.13 & 7990.19 \\
Acetone & 1.35 & 0.63 & 0.79 & 26184.81 & 8605.97 \\
DCM & 1.42 & 0.59 & 0.58 & 5519.84 & 27090.82 \\
THF & 1.78 & 0.54 & 0.54 & 7549.7 & 25898.74 \\
EA & 1.37 & 0.48 & 0.99 & 5252.1 & 27135.85 \\
\hline
\end{tabular}


DHBA is weakly soluble in less polar solvents. A blue shift in absorption spectra with increasing solvent polarity for DHBA is observed while the emission spectra consists three fluorescence component at (1) 405$415 \mathrm{~nm}$, (2) 430-450nm, and (3) 450$470 \mathrm{~nm} .(1)$ and (3) appear in less polar solvents while (2) appears in polar solvents. The (1) is the usual monomeric fluorescence of DHBA in neutral form that is stable under the non-polar environment, however because carboxyl group of DHBA is close to its neighbouring hydroxyl group and $\mathrm{pKa}$ of hydroxy group in the excited state is so called phototautomerism takes place in a DHBA molecule. The proton of 2-hydroxy group of DHBA is considered to be released and to be captured by 1-carboxy group giving birth to a zwitterions of DHBA. The fluorescence can be assigned to that of the zwitterions. On the other hand, 5-hydroxy group of DHBA cannot get carboxyl group paired. In less polar solvents, 5-hydroxy group is stable as its neutral state even if it is irradiated. The steady state spectral parameter of DHBA in different solvents are given in table.

However in polar solvents, the proton of 5hydroxy group is released even in the ground state, through the fraction is not much, thus the fluorescence (2) is of anionic DHBA. The absorption spectra of DHBA have been recorded in a series of alcohol. Concentration of the solute has been kept constant at $10^{-4} \mathrm{M}$ for all measurements. No change in shape of absorption and emission spectra has been observed. A remarkable response of DHBA towards viscosity of the medium has been observed. There is a red shift in absorption spectra while going to higher alcohol (328nm$335 \mathrm{~nm})$. The fluorescence emission spectra of DHBA show sensitivity to the solvent viscosity, with increasing solvent viscosity (octanol to methanol ). There is a red shift in absorption spectra while going to higher alcohol. The concentration of DHBA was kept at $1 \times 10^{-4} \mathrm{~mol} / \mathrm{L}$. in solvent study. Dielectric constant, stoke shift, solvent polarity function $F_{1}$ and $F_{2}$. Spectral shifts $\left(v_{f}-v_{a}\right)$ versus solvent polarity function $F_{1}$ for various solvents is shown in Fig.7. The linear progression is done by origin 6 software and the data fitted to the straight line. We have obtained high value of correlation coefficient with large number of data points which indicates good linearity with all the slope. The deviation from linearity may occur due to specific solutesolvent interaction.

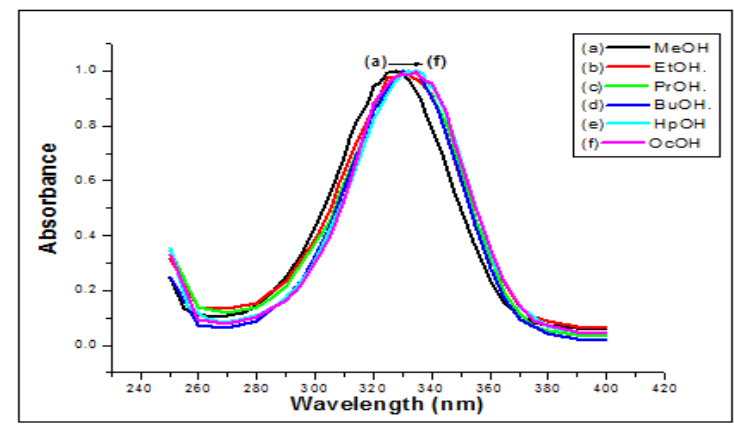

Fig 4 -Absorbance spectra in different a kohols

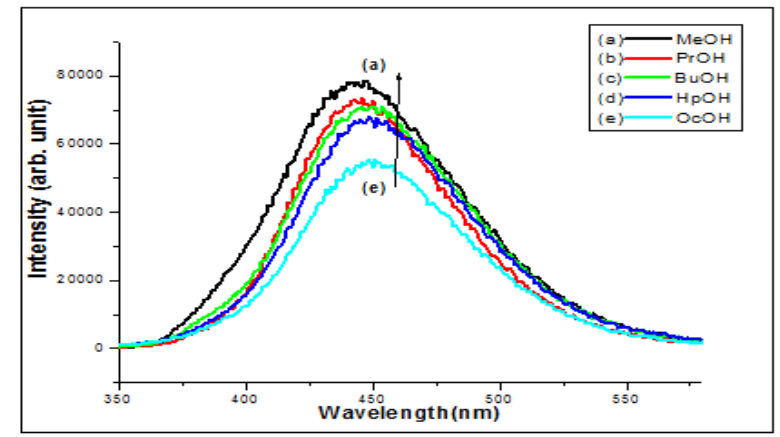

Fig. 5- Emission spectra of 2, 5-DHBA in a lcohol 


\section{Conclusions}

On summarization of above re-investigation indicates that substitution of hydroxyl and carboxylic acid at different

position of benzene ring shows marked change in the photo physics of benzoic acid. Different ionic species were identified by addition of polar, aprotic and non polar solvents. Investigation of DHBA in polar, aprotic and non polar has been carried out on the spectral parameter. Absorption and emission maxima of DHBA in different alcohols are found to be affected with respect to viscosity of alcohols. There is a red shift in absorption and emission spectra while going to higher alcohols. Role of functional group of DHBA in different solvent have been demonstrated in this work.

\section{Reference}

[1] C.Reichardt, VCH Publishers: Weinheim (1998)

[2] P. Suppan, J. Of Photochem. And Photobio. A, 50 (0990) 293.

[3] L.V. Natarajan, R.S.Becker, Macromolecules, 21(1988) 73.

[4] J.P. Bridhkoti, R. Gahlaut, H.C. Joshi, S. Pant, J. Lumin. 131 (2011) 1869.

[5] N. Tewari, N.K. Joshi, R. Rautela, R. Gahlaut, H.C. Joshi, S. Pant, J. Mol. Liq. 16 (2011) 150.

[6] R. Rautela, N.K. Joshi, H.C. Joshi, S. Pant, J. Mol. Liq. 157 (2010) 162. 